\title{
Effect of Different Doses of Paracetamol on Postoperative Pain After Gynecologic Laparoscopy
}

\author{
Simin Atashkhoei ${ }^{1}$, Fariba Nikan $^{2}, \operatorname{Reza} \operatorname{Kardan}^{2}$, Hojjat Pourfathi ${ }^{2 *}(\mathbb{D}$
}

\begin{abstract}
Objectives: This study aimed to investigate the analgesic efficacy and safety of preventive administration of $2 \mathrm{~g}$ of paracetamol compared with $1 \mathrm{~g}$ for the management of postoperative pain in the patients undergoing gynecologic laparoscopic procedures. Methods and Materials: This double-blind study was conducted on 92 women who were randomly assigned into two groups: paracetamol $2 \mathrm{~g}$ (study group; $\mathrm{n}=46$ ) and $1 \mathrm{~g}$ (control group; $\mathrm{n}=46$ ) into $100 \mathrm{~mL}$ normal saline, infused over 15 minutes in the end of surgery. Abdominal and shoulder pain scores were recorded in post-anesthesia care unit (PACU), 1, 2, 3, 6, 12, and 24 hours after the operation. The time of first request for analgesic and the values of liver enzymes were recorded.

Results: During 24 hours after surgery, the prevalence of postoperative abdominal pain was $52.17 \%$ and $89.13 \%(P<0.001)$ and shoulder pain was $6.52 \%$ and $23.91 \%(P=0.039)$ in the study and control groups, respectively. Abdominal pain score $(0.06 \pm 0.32$ vs. $1.6 \pm 2.0 ; P<0.001)$ and shoulder pain score $(0.0 \pm 0.0$ vs. $0.50 \pm 1.37 ; P=0.017)$ in PACU were lower in the study group compared to the control group. The time to first request for analgesic was significantly longer in the study group than that in the control group $(P=$ $0.030)$. There was no significant difference in liver enzyme values in postoperative 24 hours between the groups $(P>0.05)$.

Conclusions: Administration of both doses of paracetamol at the end of surgery was effective on postoperative pain; however, the best pain relief was obtained by paracetamol $2 \mathrm{~g}$ with no side effects.

Keywords: Gynecologic surgery, Laparoscopy, Postoperative pain, Paracetamol
\end{abstract}

\section{Introduction}

Laparoscopic surgery is a minimally invasive technique associated with less postoperative pain. However, laparoscopic procedures are associated with moderate to severe postoperative pain, frequently in abdomen or shoulder regions in most patients, particularly on the first postoperative day. Studies show that $80 \%$ of patients require systemic opioid analgesia after laparoscopic surgery $(1,2)$.

Different approaches have been recommended for the treatment of postoperative pain. Systemic, local, and neuraxial medications (as preemptive, preventive, or postoperative administration) are the commonly used modalities for postoperative pain relief (3-5). However, they may not completely relieve postoperative pain, and/ or have the potential for debilitation and serious adverse reactions (3-8). Paracetamol is used as a supplemental analgesic or single modality to reduce postoperative pain. Paracetamol takes both central inhibitor action on cyclooxygenases (cox-3) and interaction with the serotonergic system. In addition, paracetamol is a weak cox-1 and cox-2 inhibitor (anti-inflammatory effect). Moreover, paracetamol does not have the adverse effects of nonsteroidal anti-inflammatory drugs (NSAIDs) or opioids (9-12).
Paracetamol (intravenous acetaminophen) is a nonopioid analgesic which is devoid of risks related to opioids (10). The usage of paracetamol after various surgical procedures in decreasing acute pain has been shown $(11,12)$. The mechanism of action is not completely understood; it is thought to act through the inhibition of prostaglandin synthetase in the CNS (13). The combination of paracetamol with other analgesics working on different pain mechanisms may be an improvement in postoperative analgesia and reduction of side effects (9). The recommended dose for paracetamol in adults is $1 \mathrm{~g}$, which can be administered every 6 hours per day (14).

There are conflicting results concerning the analgesic effect of paracetamol $1 \mathrm{~g}$ in postoperative pain control, especially severe pain. Likewise, there is limited information on using a high starting dose of paracetamol for postoperative pain control (15-17). In addition, no other study on the preventive administration of larger doses of paracetamol for the management of laparoscopic pain is available. In a previous study, we found advantages of administration of $1 \mathrm{~g}$ of paracetamol at the end of surgery (preventive analgesia) in the patients undergoing cesarean section (18). Thus, this study was designed to evaluate the analgesic efficacy and opioid-sparing activity of $2 \mathrm{~g}$ of paracetamol compared with $1 \mathrm{~g}$ at the end of

Received 9 March 2017, Accepted 14 December 2017, Available online 2 January 2018

${ }^{1}$ Department of Anesthesiology, Al-Zahra Hospital, Tabriz University of Medical Sciences, Tabriz, Iran. ${ }^{2}$ Department of Anesthesiology, Taleghani Hospital, Tabriz University of Medical Sciences, Tabriz, Iran

*Corresponding Author: Hojjat Pourfathi, Tel: +989143138969, Email: hojjatpourfath@yahoo.com 
operation (preventive analgesia) in the patients who had undergone laparoscopic gynecologic surgery.

\section{Materials and Methods}

This randomized controlled trial was done in the Alzahra Research and Training Center of Tabriz University of Medical Sciences from October 2014 to September 2015. This study was a double-blind clinical trial, because both patients and assessor were unaware of the data, administered medication, and type of injected medicine in all the steps. Medicines were prepared by the hospital pharmacy in identical appearance (in similar syringes) and coded. The coding remained blinded until the end of the study. At the same time, the person who cared for the patients in the postoperative period and assessed the postoperative outcomes was blinded regarding the specific group each patient belonged to. Operations were performed by two laparoscopists. Two anesthesiologists were responsible for monitoring the anesthesia and postoperative management.

Sample of Study, Sample Size and Sampling Method Women with American Society of Anesthesiologists classification (ASA) I or II, aged between 20 and 70, who had indication for laparoscopic gynecology and referred for medical treatment were enrolled through the random sampling method. The process of randomizing the patients in two groups was conducted using online software (https://www.graphpad.com/quickcalcs/randomize1. cfm). The participants were examined carefully in terms of inclusion and exclusion criteria. Comprehensive information on the objectives of the study, its benefits, confidentiality of the collected data, and the procedure of the study was provided to them. All eligible subjects were enrolled in this study and asked to complete informed consent form. Then they were randomly assigned into two groups. These two groups were distinguished according to proprietary code such that neither of the participants in the study nor the assessors and recorders of variables were informed of actual grouping.

\section{Sample Size}

Considering our previous study (15), we determined that an effective sample size of $n=84$ (42 per group) would be required for the current study to provide statistical power of $80 \%$ (two-tailed, $\alpha=0.05$ ) to detect a mean difference between the two groups, so: $n=[(Z 1-\alpha / 2+Z 1-\beta) 2 \times($ SD12 + SD22] / (Mean1 - Mean2)2. Considering 10\% dropouts of samples, a total of 92 women were recruited to take part in this study and all of them remained till the end of the study and consented to proceed with the study protocol. All eligible women were randomly assigned, on 1:1 ratio, to the study and control groups: paracetamol $2 \mathrm{~g}$ (study group; $\mathrm{n}=46$ ) or paracetamol $1 \mathrm{~g}$ (control group; $\mathrm{n}=46$ ) into $100 \mathrm{~mL}$ of normal saline over 15 minutes infused before the end of procedure (operators of the study were not involved in the subjects' enrolment and assignment to the groups).

\section{Inclusion Criteria}

- $\quad$ ASA physical status I or II female patients

- $\quad$ aged between 20 and 70

- candidate for laparoscopy

Exclusion criteria

- Allergy to paracetamol

- Chronic pain syndromes

- Pregnancy

- History of psychotic diseases

- History of systemic dysfunction of organs (cardiovascular, renal, ...)

- Laparotomy with laparoscopy

- Usage of sedative

- Hepatic disorders

- $\quad B M I \geq 35 \mathrm{~kg} / \mathrm{m}^{2}$

Patients were premeditated with oral diazepam $5 \mathrm{mg}, 1$ hour before operation. Anesthesia was standardized and induced with midazolam $0.05 \mathrm{mg} / \mathrm{kg}$, remifentanil $1-2$ $\mu \mathrm{g} / \mathrm{kg}$, and propofol $1.2-5 \mathrm{mg} / \mathrm{kg}$ and atracurium $0.5 \mathrm{mg} /$ $\mathrm{kg}$, and then intubated. Anesthesia was maintained with the infusion of propofol (50-100 $\mu \mathrm{g} / \mathrm{kg}$ ), and remifentanil (half of primary dose) if needed. Paracetamol $2 \mathrm{~g}$ (study group, $\mathrm{n}=46$ ) or $1 \mathrm{~g}$ (control group; $\mathrm{n}=46$ ) into $100 \mathrm{~mL}$ normal saline was infused over 15 minutes in the end of surgery. When the patients were awake and tracheally extubated, they were transferred to post-anesthesia care unit (PACU) and controlled, and their vital signs were recorded and assessed, and the intensity of postoperative abdominal and shoulder pain was measured by a $10-\mathrm{cm}$ linear visual analogue scale (VAS), 1, 2, 3, 6, 12, and 24 hours after operation. The patients were familiarized with the scale preoperatively. Meperidine $(0.1-5 \mathrm{mg} / \mathrm{kg}$ ) was administrated to the patients with VAS $\geq 4$ and sodium diclofenac suppository $(100 \mathrm{mg})$ for VAS $<4$. Time of first consumption of postoperative analgesics, and side effects (nausea/vomiting, sedation, dizziness, shivering, and uncomfortability) during the postoperative 24 hours were recorded. Postoperative nausea and vomiting (PONV) were treated by metoclopramide $5 \mathrm{mg}$. The safety of paracetamol $2 \mathrm{~g}$ was assessed via laboratory assessments of liver function measurements (SGOT [AST], SGPT [ALT], $\mathrm{LDH}$, and ALP). The measurement of liver enzymes was performed 24 hours after the operation.

\section{Statistical Analysis}

Demographic data, causes of laparoscopy, and duration of surgery and anesthesia were compared between two groups by the independent $t$ test, Fisher exact test, chi-square test, Mann-Whitney $U$ test and PearsonMonte Carlo. All analyses were performed using SPSS 23.00 statistical software. $P$ value $<0.05$ was considered statistically significant. 


\section{Results}

All eligible patients were willing to participate in the study, and no one refused to participate. A total of 92 women enrolled in the study. Demographic data (age, weight, and height), causes of laparoscopy, and duration of operation and anesthesia were compared. There was not significant difference between them $(P>0.05)$ (Table 1$)$.

Postoperative pain scores are presented in Figure 1. The mean abdominal pain score in the study group was significantly lower than that in the control group in the PACU and at 1 hour after operation $(P<0.001)$. Mean shoulder pain scores were also significantly lower in the study group in the PACU $(P<0.001)$, but this was not significantly different for other times $(P>0.05)$.

The number of patients without abdominal pain $(47.83 \%$ vs. $10.87 \% ; P<0.001)$ and shoulder pain $(93.48 \%$ vs. $76.09 \% ; P=0.039)$ was lower in the study group compared to the control group during the first day of operation. Table 2 shows other postoperative parameters.
The time of first request for analgesia in the study group was significantly higher than that in the control group (3.6 \pm 3.6 vs. $2.3 \pm 3.1$, respectively) $(P=0.030)$ (Table 2 ).

The study group used significantly less meperidine $(P=0.002)$ and diclofenac suppository $(P=0.013)$ than the control group over 24 hours.

The safety of paracetamol was assessed by measuring liver enzymes in the two groups, and there was no problem. There was also no clinically significant difference between the groups in terms of other side effects (Table 2).

\section{Discussion}

This study investigated the effect of different doses of paracetamol on postoperative pain after gynecologic laparoscopy. Our results showed during 24 hours after surgery, the prevalence of postoperative abdominal and shoulder pain in the study group was significantly lower compared to the control group. And abdominal and shoulder pain scores were lower in the study group

Table 1. Demographic, Surgical and Anesthesia Data $(n=92)$

\begin{tabular}{|c|c|c|c|}
\hline & Study Group $(n=46)$ & Control Group $(n=46)$ & $P$ Value \\
\hline & Mean (SD) & Mean (SD) & \\
\hline Age $(y)^{a}$ & 30.5 (8.9) & $31.8(5.9)$ & 0.159 \\
\hline Weight (kg) ${ }^{\mathrm{a}}$ & $70.2(11.5)$ & $66.1(9.9)$ & 0.076 \\
\hline Height $(\mathrm{cm})^{\mathrm{a}}$ & $160.3(5.9)$ & 160.7 (3. 7) & 0.764 \\
\hline BMI $\left(\mathrm{kg} / \mathrm{m}^{2}\right)^{\mathrm{a}}$ & $27.2(4.1)$ & $25.6(3.8)$ & 0.054 \\
\hline Operation time $(\mathrm{min})^{\mathrm{a}}$ & $55.2(21.8)$ & $57.3(25.6)$ & 0.810 \\
\hline Anesthesia time $(\mathrm{min})^{\mathrm{a}}$ & 71.9 (22.9) & $73.3(26.2)$ & 0.684 \\
\hline Causes of laparoscopy $(\%)^{b}$ & & & 0.264 \\
\hline Infertility & $21(45.7)$ & $26(56.6)$ & \\
\hline Ovarian cystectomy & $15(32.6)$ & $15(32.6)$ & \\
\hline Others & $10(21.7)$ & $5(10.8)$ & \\
\hline
\end{tabular}

a By independent samples $t$ test.

${ }^{b}$ By Pearson-Monte Carlo $(95 \% \mathrm{Cl})$

$P$ value $<0.05$ is significant.
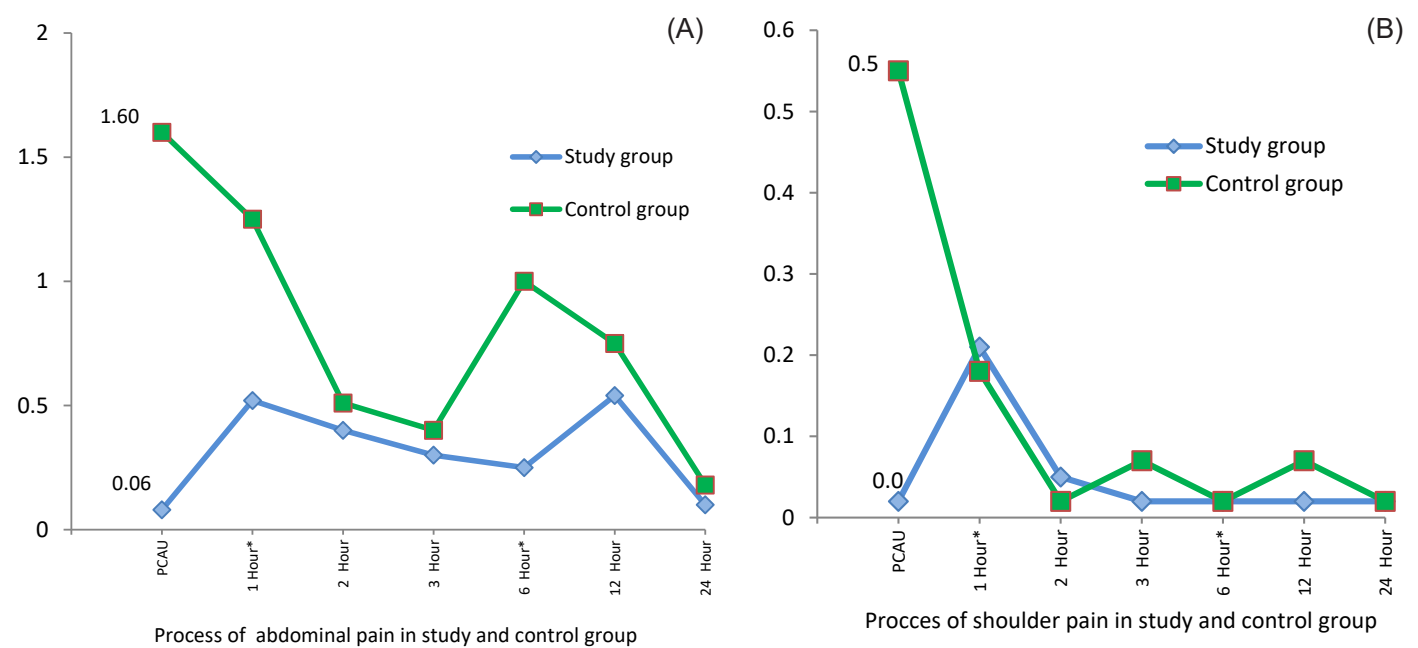

Figure 1. Visual analogues scale (Vas). Values are mean $\pm S D$. (A) Abdominal pain: $P<0.001$ in PACU and $1 \mathrm{~h}$ after operation, and $P>0.05$ in other times of postoperative. (B) Shoulder pain: $P<0.001$ in PACU, and $P>0.05$ in other times of postoperative. 
Table 2. Postoperative Parameters in 2 Groups $(n=92)$

\begin{tabular}{|c|c|c|c|}
\hline & Study Group $(n=46)$ & Control Group $(n=46)$ & $P$ Value \\
\hline Incidence of abdominal pain in PACU (\%) & $2(4.3)$ & $22(47.8)$ & $<0.001$ \\
\hline Overall incidence of abdominal pain (24 h) (\%) ${ }^{a}$ & $2(4.3)$ & $3(6.5)$ & $>0.05$ \\
\hline Incidence of shoulder pain in PACU (\%) & $0(0.0)$ & $6(13.04)$ & 0.013 \\
\hline Overall incidence of shoulder pain $(24 \mathrm{~h})(\%)^{\mathrm{b}}$ & $0(0.0)$ & $0(0.0)$ & $>0.05$ \\
\hline Time to first analgesic $(h)^{c}$ & $3.6 \pm 3.6$ & $2.3 \pm 3.1$ & 0.030 \\
\hline Patients receiving analgesia (Patients with VAS>4) (\%) & $25(54.0)$ & $40(87.0)$ & 0.001 \\
\hline Meperidine consumption (mg) & $0.2 \pm 43.9$ & $5.76 \pm 10.5$ & 0.002 \\
\hline Diclofenac suppository consumption (mg) & $71.7 \pm 112.9$ & $163.0 \pm 214.3$ & 0.013 \\
\hline \multicolumn{4}{|l|}{ Incidence of side effects (\%) } \\
\hline PONV & $4(8.6)$ & $8(17.4)$ & 0.216 \\
\hline Sedation & $1(2.2)$ & $0(0.0)$ & 1.00 \\
\hline Dizziness & $1(2.2)$ & $0(0.0)$ & 1.00 \\
\hline Shivering & $1(2.2)$ & $2(4.3)$ & 1.00 \\
\hline Uncomfortability & $2(4.3)$ & $2(4.3)$ & 0.692 \\
\hline \multicolumn{4}{|l|}{ Liver enzymes measurements (u) } \\
\hline AST (SGOT) & $17.1 \pm 4.7$ & $17.2 \pm 5.8$ & 0.937 \\
\hline ALT (SGPT) & $13.3 \pm 4.6$ & $15.1 \pm 6.1$ & 0.135 \\
\hline ALP & $139.9 \pm 36.9$ & $143.2 \pm 38.9$ & 0.681 \\
\hline LDH & $316.6 \pm 101.9$ & $297.2 \pm 92.9$ & 0.343 \\
\hline
\end{tabular}

\footnotetext{
${ }^{a}$ By chi-square test

${ }^{\mathrm{b}}$ By Fisher exact test

'By Mann-Whitney U, (U=339).

$P$ value $<0.05$ is significant.
}

compared with the control group. The time to first request for analgesic was significantly longer in the study group than that in the control group. There was no significant difference in liver enzyme values in the postoperative 24 hours between the two groups.

Adequate operative pain management is essential, and might help to reduce pain-related complications and improve postoperative outcome (1-8).

Our results showed during 24 hours after surgery, the prevalence of postoperative abdominal and shoulder pain was significantly lower compared to the control group. Juhl et al (17) evaluated the analgesic efficacy and safety of paracetamol $2 \mathrm{~g}$ in comparison with $1 \mathrm{~g}$ or placebo after molar surgery. They reported superior activity with paracetamol $2 \mathrm{~g}$ administered after surgery in terms of magnitude and duration of analgesic effect for postoperative pain, which is consistent with our study results.

A study by Silvanto et al (19) showed that administration of $3 \mathrm{~g}$ of paracetamol during the early period of tonsilectomy reduced the consumption of opioid. Furthermore, Moon et al (1) evaluated the effect of preoperative paracetamol $2 \mathrm{~g}$ compared to placebo in the patients undergoing abdominal hysterectomy. Their results showed that premedication with a high dose of paracetamol (30 minutes before the beginning of surgery) reduced opioid consumption and opioidrelated side effects, without significantly reducing pain intensity. The present study showed that premedication with paracetamol provided pain relief and postoperative analgesia requirements after laparoscopic gynecologic procedures, however paracetamol $2 \mathrm{~g}$ was more effective, which is consistent with other studies $(15,20,21)$.

Preventive analgesia was considered to be a suitable definition for both the intraoperative administration of drug and its administration at the end of the surgery for preventing postoperative pain (18). The present findings demonstrated that the injection of paracetamol prior to the end of surgery effectively suppressed all possible nociceptor transduction pathways, preventing central sensitization and improving postoperative pain management $(5,19,22)$. In our previous study, we also observed the efficacy of preventive analgesia with paracetamol $1 \mathrm{~g}$ in postoperative pain after cesarean section (18).

In this study, the safety was assessed by measurement of liver enzymes for 24 hours. Results showed that there was no hepatic effect in the patients. This dose was below the threshold of hepatotoxicity in healthy patients (1417). Silvanto et al (19) administered a single $3 \mathrm{~g}$ dose of paracetamol after tonsilectomy. They concluded that higher doses of paracetamol had approximately equal analgesic efficacy as conventional doses, although no definitive hepatotoxicity was observed in their study.

In our study, PONV was seen in $14(15.21 \%)$ patients of the two groups. However, paracetamol $2 \mathrm{~g}$ was more associated with a decreased incidence of PONV than 1 $\mathrm{g}$ was. One of the possible mechanisms is that decreased opioid requirement in paracetamol $2 \mathrm{~g}$ contributes to the decreased opioid-related side effects (19). Cok et al (23) reported that intraoperative administration of paracetamol 
decreased the incidence of PONV after strabismus surgery. In our study, we found no statistically significant difference with regard to other postoperative side effects (sedation, dizziness, shivering, and uncomfortability) between the patients of both groups; it may be because of low doses of opioids used in the postoperative period.

The limitation of our study was lack of a placebo group for comparing the effects of placebo with each of the two groups, and that all of the patients were female. Further studies are necessary in men and children. In addition, further studies with greater doses of paracetamol ( $\geq 2 \mathrm{~g}$ ) are required.

\section{Conclusions}

Administration of both doses of paracetamol over 15 minutes before the end of surgery was effective on postoperative pain. However, the best pain relief was obtained by paracetamol $2 \mathrm{~g}$, with no side effects.

\section{Conflict of Interests}

Authors declare that they have no conflict of interests.

\section{Ethical Issues}

The study protocol was approved by the Ethics Committee of Tabriz University of Medical Sciences (Code and date of ethical approval: 92187 and October 21, 2013). It was also registered in the Iranian Registry of Clinical Trials website (identifier: IRCT201312147013N5). In addition, participants were provided with the information on the study procedure. A signed consent was obtained before data collection.

\section{Financial Support}

This work was partially supported by the financial helps of Tabriz University of Medical Sciences.

\section{Acknowledgements}

We are thankful for cooperation provided by Al-Zahra Research and Training Center of Tabriz University of Medical Sciences, all personnel of this center, and all participants in this study.

\section{References}

1. Moon YE, Lee YK, Lee J, Moon DE. The effects of preoperative intravenous acetaminophen in patients undergoing abdominal hysterectomy. Arch Gynecol Obstet. 2011;284(6):1455-1460. doi:10.1007/s00404-011-1860-7

2. Miller RD. Miller's Anesthesia. 7th ed. Philadelphia: Churchill Livingstone; 2010:2758-2760.

3. Apfelbaum JL, Chen C, Mehta SS, Gan TJ. Postoperative pain experience: results from a national survey suggest postoperative pain continues to be undermanaged. Anesth Analg. 2003;97(2):534-540, table of contents.

4. Mouton WG, Bessell JR, Otten KT, Maddern GJ. Pain after laparoscopy. Surg Endosc. 1999;13(5):445-448.

5. Akinci SB, Ayhan B, Aycan IO, et al. The postoperative analgesic efficacy of intraperitoneal tramadol compared to normal saline or intravenous tramadol in laparoscopic cholecystectomy. Eur J Anaesthesiol. 2008;25(5):375-381. doi:10.1017/s0265021508003694

6. Pappas-Gogos G, Tsimogiannis KE, Zikos N, Nikas K, Manataki A, Tsimoyiannis EC. Preincisional and intraperitoneal ropivacaine plus normal saline infusion for postoperative pain relief after laparoscopic cholecystectomy: a randomized double-blind controlled trial. Surg Endosc. 2008;22(9):2036-2045. doi:10.1007/s00464-008-9762-x

7. Shapiro A, Zohar E, Hoppenstein D, Ifrach N, Jedeikin R, Fredman B. A comparison of three techniques for acute postoperative pain control following major abdominal surgery. J Clin Anesth. 2003;15(5):345-350.

8. Lavand'homme P, De Kock M, Waterloos H. Intraoperative epidural analgesia combined with ketamine provides effective preventive analgesia in patients undergoing major digestive surgery. Anesthesiology. 2005;103(4):813-820.

9. Gadsden J, Hart S, Santos AC. Post-cesarean delivery analgesia. Anesth Analg. 2005;101(5 Suppl):S62-69.

10. Cattabriga I, Pacini D, Lamazza G, et al. Intravenous paracetamol as adjunctive treatment for postoperative pain after cardiac surgery: a double blind randomized controlled trial. Eur J Cardiothorac Surg. 2007;32(3):527531. doi:10.1016/j.ejcts.2007.05.017

11. Vosoughin M, Mohammadi S, Dabbagh A. Intravenous ketamine compared with diclofenac suppository in suppressing acute postoperative pain in women undergoing gynecologic laparoscopy. J Anesth. 2012;26(5):732-737. doi:10.1007/s00540-012-1399-1

12. Coulthard P, Hill CM, Frame JW, Barry H, Ridge BD, Bacon TH. Pain control with paracetamol from a sustained release formulation and a standard release formulation after third molar surgery: a randomised controlled trial. Br Dent J. 2001;191(6):319-324. doi:10.1038/sj.bdj.4801174a

13. Rang HP, Dale MM, Ritter JM. Anti-inflammatory and immunosuppresant drugs. In: Pharmacology Fourth Edition. Edinburgh: Churchill Livingstone; 1999:229-247.

14. Uysal HY, Takmaz SA, Yaman F, Baltaci B, Basar H. The efficacy of intravenous paracetamol versus tramadol for postoperative analgesia after adenotonsillectomy in children. J Clin Anesth. 2011;23(1):53-57. doi:10.1016/j. jclinane.2010.07.001

15. Wininger SJ, Miller H, Minkowitz HS, et al. A randomized, double-blind, placebo-controlled, multicenter, repeat-dose study of two intravenous acetaminophen dosing regimens for the treatment of pain after abdominal laparoscopic surgery. Clin Ther. 2010;32(14):2348-2369. doi:10.1016/j. clinthera.2010.12.011

16. Remy C, Marret E, Bonnet F. State of the art of paracetamol in acute pain therapy. Curr Opin Anaesthesiol. 2006;19(5):562565. doi:10.1097/01.aco.0000245285.30282.70

17. Juhl GI, Norholt SE, Tonnesen E, Hiesse-Provost O, Jensen TS. Analgesic efficacy and safety of intravenous paracetamol (acetaminophen) administered as a $2 \mathrm{~g}$ starting dose following third molar surgery. Eur J Pain. 2006;10(4):371377. doi:10.1016/j.ejpain.2005.06.004

18. Atashkhoyi S, Rasouli , Fardiazar Z, Ghojazadeh M, Hatami Marandi P. Preventive Analgesia with Intravenous Paracetamol for Post-cesarean section Pain Control. Int J Womens Health Reprod Sci. 2014;2(3):131-137. doi:10.15296/ijwhr.2014.20 
19. Silvanto M, Musterhjelm E, Savolainen S, et al. Effect of 3 $\mathrm{g}$ of intravenous paracetamol on post-operative analgesia, platelet function and liver enzymes in patients undergoing tonsillectomy under local anaesthesia. Acta Anaesthesiol Scand. 2007;51(9):1147-1154. doi:10.1111/j.13996576.2007.01376.x

20. Inal M, Celik N, Tuncay F. I.V. Paracetamol Infusion Is Better Than I.V. Meperidine Infusion For Postoperative Analgesia After Caesarean Section. The Internet Journal of Anesthesiology. 2006;15(1):1-6.

21. Serinken M, Eken C, Turkcuer I, Elicabuk H, Uyanik $\mathrm{E}$, Schultz $\mathrm{CH}$. Intravenous paracetamol versus morphine for renal colic in the emergency department: a randomised double-blind controlled trial. Emerg Med J. 2012;29(11):902-905. doi:10.1136/emermed-2011-200165

22. Maund E, McDaid C, Rice S, Wright K, Jenkins B, Woolacott N. Paracetamol and selective and non-selective non-steroidal anti-inflammatory drugs for the reduction in morphine-related side-effects after major surgery: a systematic review. Br J Anaesth. 2011;106(3):292-297. doi:10.1093/bja/aeq406

23. Cok OY, Eker HE, Pelit A, et al. The effect of paracetamol on postoperative nausea and vomiting during the first 24 h after strabismus surgery: a prospective, randomised, double-blind study. Eur J Anaesthesiol. 2011;28(12):836841. doi:10.1097/EJA.0b013e32834c580b

(C) 2018 The Author (s); This is an open-access article distributed under the terms of the Creative Commons Attribution License (http://creativecommons.org/licenses/by/4.0), which permits unrestricted use, distribution, and reproduction in any medium, provided the original work is properly cited. 